\title{
All-Optical Tunable Delay With NRZ-to-RZ Format Conversion Capability Based on Optical Kerr Switch and Pulse Pre-Chirping
}

\author{
Bill Ping-Piu Kuo, Student Member, IEEE, P. C. Chui, and Kenneth Kin-Yip Wong, Member, IEEE, Member, OSA
}

\begin{abstract}
All-optical tunable delay plays an important role for enabling high-throughput optical routing, such as buffer for contention resolution and timing synchronization. We proposed and demonstrated a widely tunable optical delay using optical Kerr-switch-based wavelength converter and pulse pre-chirping for compensating chromatic dispersion in fiber delay. With wideband operation provided by a Kerr-switch wavelength converter, a tunable delay range up to $8.56 \mathrm{~ns}$ was achieved. Meanwhile, pulse pre-chirping helped to alleviate group-velocity dispersion (GVD)-induced penalty, and provided simultaneous nonreturn-zero (NRZ)-to-RZ format conversion functionality.
\end{abstract}

Index Terms-Cross-polarization modulation, dispersion compensation, optical delay.

\section{INTRODUCTION}

$\mathbf{I}$ N THE context of optical signal processing, optically controllable tunable delay has been an important aspect for its significant in enabling all-optical routing and packet processing in optical network, and for phase control and beam forming in photonic microwave signal processing [1], [2]. In particular, fiber-based techniques have additional advantages due to the availability of low-cost components. Previously, fiber-based delay devices have been realized by using gain-induced group-velocity modulation based on Brillouin [3], Raman [4] and optical parametric process [5], [6]. However, these approaches rely on steep gain slope and induced dispersion of the amplifiers and thus would result in severe pulse distortion [7]. Another design philosophy aiming to increase usable delay of the device is to combine the group velocity dependency on wavelength of highly dispersive fiber and wavelength conversion techniques, such as self-phase modulation (SPM) [8] and idler generation in optical parametric process [9]-[13]. Unfortunately for SPM-based device, its performance depends on the wavelength detune of converted signal from the input signal and extensive pulse broadening could prohibit its use in

Manuscript received July 29, 2008. Current version published January 28, 2009. This work was supported in part by the Research Grants Council of the Hong Kong Special Administrative Region, China, under Projects HKU 7179/06E and HKU 7172/07E.

The authors are with the Photonic Systems Research Laboratory, Department of Electrical and Electronic Engineering, University of Hong Kong, Hong Kong (e-mail: kywong@eee.hku.hk).

Color versions of one or more of the figures in this paper are available online at http://ieeexplore.iee.org.

Digital Object Identifier 10.1109/JLT.2008.2004928 large delay; for OPA-based device, the input wavelength range could be limited due to stringent phase-matching condition.

In order to achieve large delay over wideband, a wavelength-insensitive approach with robustness to group-velocity dispersion (GVD) would be desirable. An optical tunable delay using optical Kerr switch for wavelength conversion and pulse pre-chirp to combat for GVD-induced distortion would be promising under these considerations [14]. Thanks to regenerative nature of Kerr switch, this approach can help to reduce pattern-dependent amplitude fluctuation due to GVD and can be used to implement wideband large delay device. Furthermore, pulse pre-chirping can be used to perform nonreturn-zero (NRZ) to return-zero (RZ) conversion through pulse compression effect [15]. As RZ format has been widely used owing to its robustness to nonlinear effect-induced signal degradation such as four-wave mixing effect in transmission fibers and optical parametric amplifiers [16]-[18], an optical delay with format conversion functionality would be desirable for use in synchronizing and bridging short-haul NRZ bit stream to RZ bit stream in long-haul links.

In this paper, we present a comprehensive study on the theory and performance of the optical tunable delay we proposed previously [19]. The organization of this paper is as follow. Section II describes the principle behind the proposed scheme, with emphases on the theory of Kerr switch and pulse pre-chirping. Section III provides details on the experimental setup used to verify the proposed scheme, and discussions on the results obtained. Concluding remark is given in Section IV.

\section{THEORY OF OPERATION}

The operating principle of the proposed optical tunable delay is based on optical Kerr switching for wideband wavelength conversion, and pulse pre-chirping to compensate for the dispersion penalty in fiber, as shown in Fig. 1. The intensity modulation of the incoming signal at $\lambda_{s}$ is transferred to a phase-modulated $\mathrm{cw}$ probe with arbitrarily chosen wavelength $\lambda_{p}$ in the first-stage Kerr switch. The converted probe is then launched to a dispersive fiber. The propagation delay of the probe wave inside the dispersive fiber depends on the wavelength of the probe due to large chromatic dispersion. Therefore, the delay of the probe can be controlled by tuning the wavelength of the probe. The delayed probe was then back converted to signal wavelength $\left(\lambda_{s}\right)$ in the second-stage Kerr switch. Detailed descriptions are provided in the following sections: 


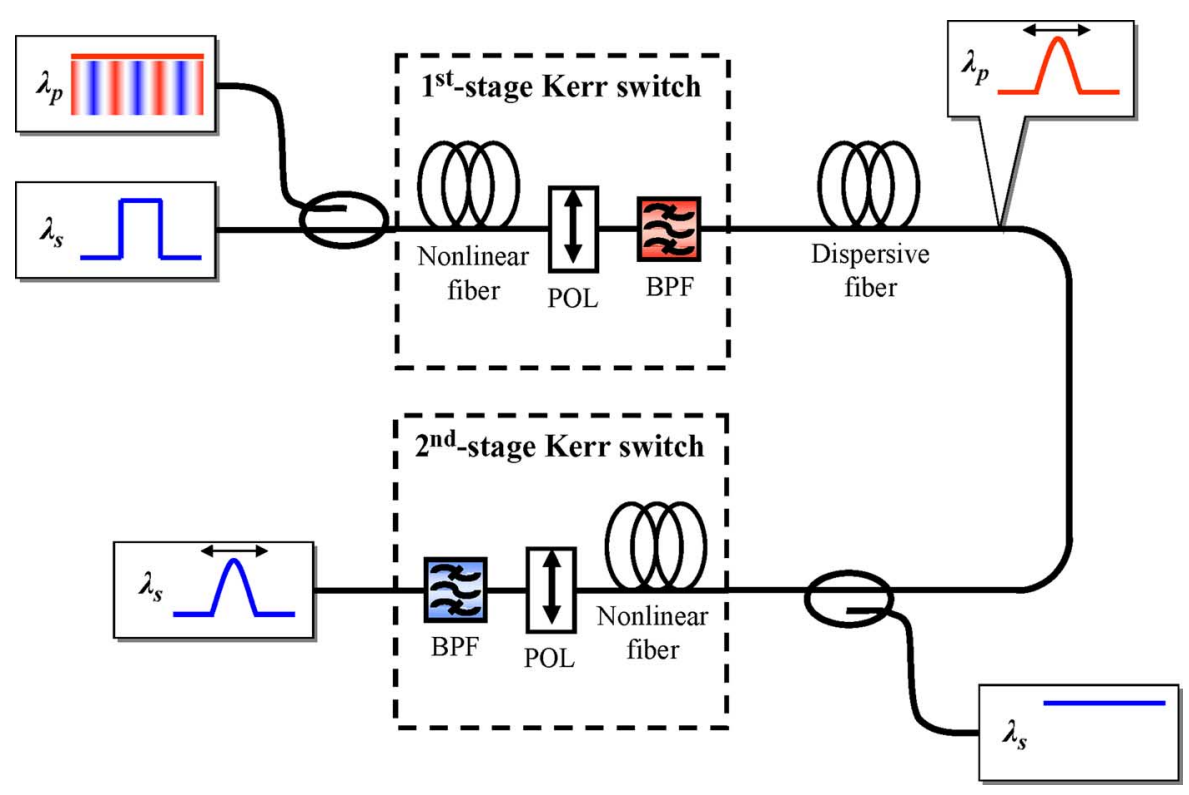

Fig. 1. Principle of Kerr switch-based optical tunable delay. POL: Polarizer; BPF: Bandpass filter. Refer to text for detailed description.

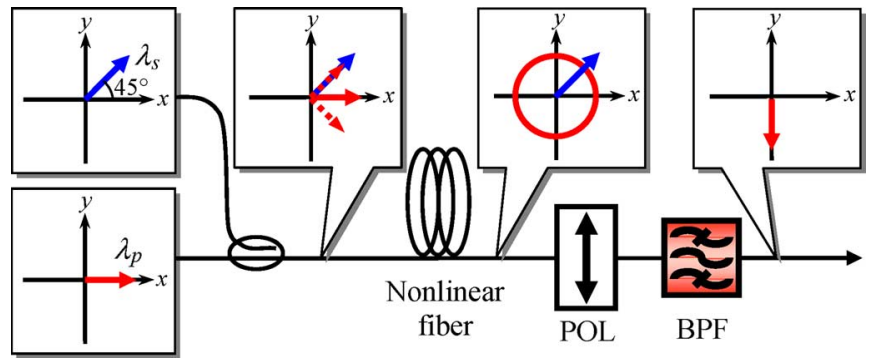

Fig. 2. Principle of Kerr switch based wavelength converter. The transmission axis of the polarizar (POL) is along $y$ axis in the polarization chart.

\section{A. Optical Kerr-Switch-Based Wavelength Conversion}

The wavelength converters in the proposed tunable delay are based on cross-polarization modulation (XPolM) in nonlinear medium. Each stage of the Kerr switch, as shown in Fig. 2, consists of a spool of nonlinear fiber for XPolM, a polarizer to convert polarization modulation to intensity modulation, and a bandpass filter to eliminate the control lightwave. At the fiber input, the intensity-modulated signal $\left(\lambda_{s}\right)$ serves as the control, which its modulation is to be transferred to another wavelength designated by a cw probe $\left(\lambda_{p}\right)$. Note that the state of polarizations (SOPs) of the input control and probe are assumed to be linear for simplicity. The SOP of the input probe is adjusted to be either orthogonal or parallel to the transmission axis of the polarizer, corresponding to noninverting or inverting mode of operation of the Kerr switch. On the other hand, the SOP of the control signal is rotated from that of probe by $45^{\circ}$. When the probe co-propagates in the nonlinear fiber, the high intensity control pulse will cause phase shift of the probe component parallel to the control due to optical Kerr effect [14], [20]. As a result, the SOP of the probe is rotated and therefore part of its energy can pass through the polarizer. Assume the power of the probe is weak so that the polarization of the control signal is not affected by the Kerr effect due to probe wave, and other

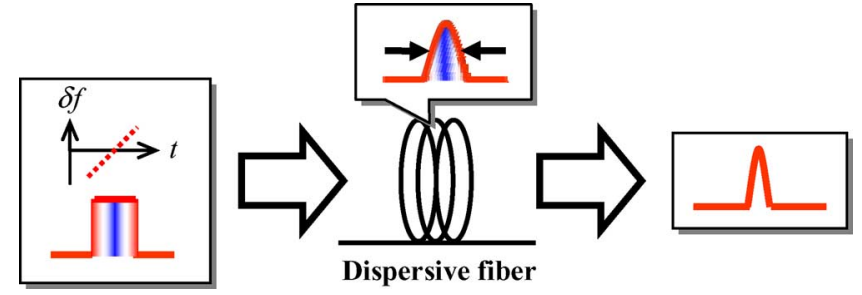

Fig. 3. Principle of GVD-compensation by pulse pre-chirping.

nonlinear effect is negligible, the transmitted power $P_{P}$ of probe wave is governed by the following function [20]:

$$
P_{P}=P_{P 0} \sin ^{2}\left(\frac{2}{3} \gamma P_{s} L\right)
$$

where $\gamma$ and $L$ are the nonlinear coefficient and length of the fiber, and $P_{s}$ is the power of the control signal respectively. From (1), if the contrast of the control signal is large enough and the power level of the mark level is close to $3 \pi / 4 \gamma L$, the intensity noise on the control signal will be suppressed when the intensity modulation is transferred to probe. It gives rise to regenerative effect of a Kerr switch.

\section{B. Pulse Pre-Chirp for Dispersion Compensation}

Although Kerr switch wavelength conversion helps to achieve large delay range with its wideband operation, dispersion-incurred penalty of the converted signal is unavoidable without special care due to its nonphase-preserving nature. To compensate for the dispersive effect, a simple pulse pre-chirp technique is deployed in this proposed scheme. Fig. 3 illustrates the principle of the pre-chirping technique. Instead of launching an unmodulated probe, the cw probe is phase-modulated with a periodic parabolic signal which is synchronized to the incoming bit stream. The parabolic phase profile of the probe wave generates a linear frequency chirp. When the chirped probe wave 


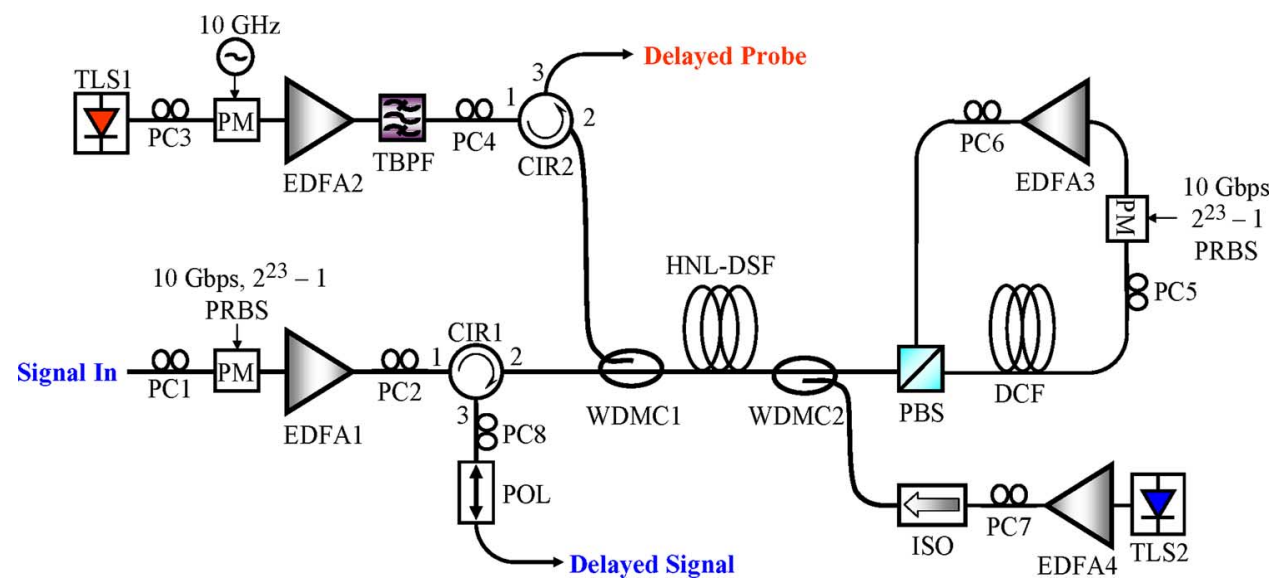

Fig. 4. Experimental setup of the proposed delay line. Refer to the text for detailed description.

propagates through fiber with large dispersion, the frequency chirp causes the pulse to be compressed temporally [15], [21]. In effect GVD-induced pulse broadening can be minimized, and at the same time, the pulse compression converts the format of the signal from NRZ to RZ. However, a parabolic signal occupies infinitely large bandwidth as it is discontinuous at the bit slot boundary, and therefore it is unrealizable in practice. Here we adopt a sinusoidal pre-chirping waveform which the trough of the waveform is aligned to the center of the bit slot. With even-symmetric phase profile, the frequency chirp is quasi-linear about the bit slot center and thereby is useful for GVD-compensation.

Note that the bit rate supported by the pre-chirp scheme depends on the total dispersion in the optical delay. Using the empirical formula provided in [21], one can deduce that the bit rate $B$ scales with total dispersion $D$ and RZ duty ratio as follow:

$$
\begin{aligned}
& 33 \% \text { duty ratio }: B \approx 0.279 \sqrt{\frac{2 \pi c}{\lambda^{2}|D|}} \\
& 50 \% \text { duty ratio }: B \approx 0.54 \sqrt{\frac{2 \pi c}{\lambda^{2}|D|}} .
\end{aligned}
$$

\section{EXPERIMENT AND DISCUSSIONS}

The experimental setup of the proposed optical tunable delay and format converter is shown in Fig. 4. In the first stage Kerr switch, the incoming NRZ signal at $1570 \mathrm{~nm}$ with intensity modulation carrying $10 \mathrm{~Gb} / \mathrm{s} 2^{31}-1$ pseudorandom binary sequence (PRBS) was phase-modulated with $10 \mathrm{~Gb} / \mathrm{s} 2^{23}-1$ PRBS for stimulated Brillouin scattering (SBS) suppression in fibers. Note that SBS suppression through phase modulation can be avoided by using fiber with high nonlinearity and SBS threshold such as bismuth-oxide fiber [22].The probe wave, on the other hand, was generated by a tunable laser source TLS1 and phase-modulated by $10 \mathrm{GHz}$ sinusoidal signal synchronized to the bit stream of the signal. The phase-modulated signal and probe were then amplified to $19 \mathrm{dBm}$ and $13 \mathrm{dBm}$, respectively, and combined using a WDM band coupler (WDMC1). The combined lightwaves were launched into a spool of $1 \mathrm{~km}$ highly-nonlinear dispersion-shifted fiber

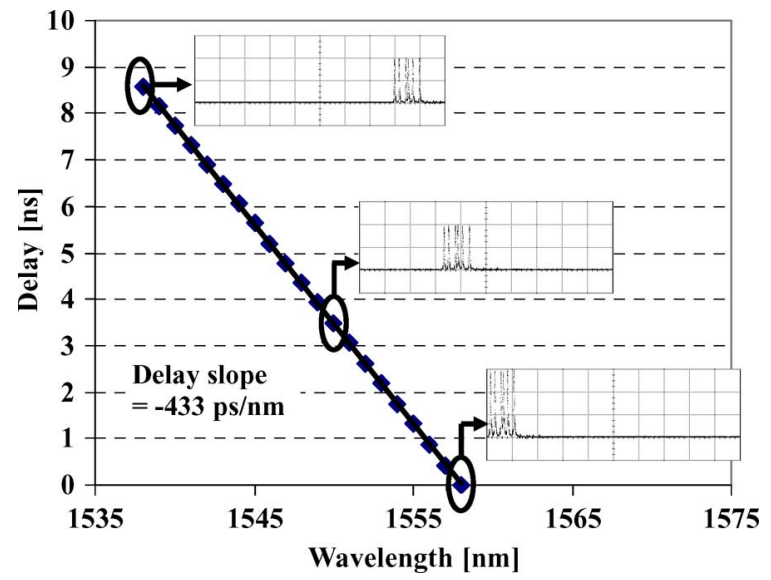

Fig. 5. Measured delay of output signal versus probe wavelength. Insets show the waveform of output signal with an input packet carrying bit pattern [101001101001]. Horizontal scale of the insets is $1.1 \mathrm{~ns} / \mathrm{div}$.

(HNL-DSF) with nonlinear coefficient $\gamma=14 / \mathrm{W} / \mathrm{km}$, zero-dispersion wavelength $\lambda_{0}=1560 \mathrm{~nm}$ and dispersion slope $d D / d \lambda=0.035 \mathrm{ps} / \mathrm{nm}^{2} \mathrm{~km}$. After XPolM in HNL-DSF, the signal and probe were decoupled using another WDM band coupler (WDMC2). The probe wave polarized by a polarization beam splitter (PBS) was launched into a spool of dispersion compensating fiber (DCF) with total dispersion of $-433 \mathrm{ps} / \mathrm{nm}$. After passing through DCF, the probe was phase dithered by $10 \mathrm{~Gb} / \mathrm{s} 2^{23}-1$ PRBS to suppress SBS in HNL-DSF, and amplified to $22 \mathrm{dBm}$ by EDFA3. The amplified probe was coupled back to WDMC2 together with the amplified CW signal at $1570 \mathrm{~nm}$ from TLS2 and then launched back into HNL-DSF for second-stage Kerr switch. The delayed signal was then retrieved at port 3 of signal circulator CIR1, polarized using polarizer POL and monitored through a digital communication analyzer (DCA) or a bit-error rate tester (BERT).

Fig. 5 shows the measured delay of the output signal against the wavelength of the probe wave. The delay of the output signal is measured relative to the minimum delay of the output signal, which is obtained when probe wavelength is $1558 \mathrm{~nm}$. From the plot, the delay of the output signal varies linearly with the probe wavelength, with slope equals to the total dispersion of the DCF used $(D \approx-433 \mathrm{ps} / \mathrm{nm})$. The maximum delay attained 


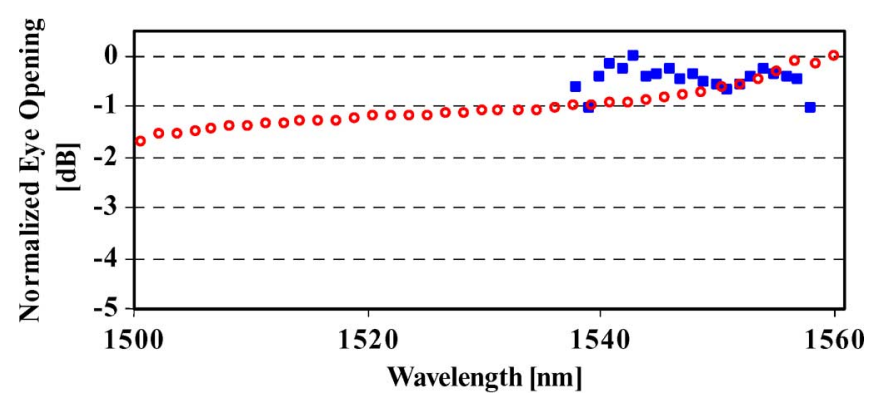

Fig. 6. Simulated (red circle) and experimental (blue square) eye opening of the converted probe. The eye opening values are normalized to the maxima of the respective data sets.

is $8.56 \mathrm{~ns}$ when probe is at $1538 \mathrm{~nm}$. The delay is limited by the gain bandwidth of the C-band EDFA (1538 nm-1565 nm). To examine the feasible wavelength range of the Kerr switch wavelength converter, a simulation was done using the same conditions as in the experiment. Fig. 6 shows the simulated and experimental eye opening (in decibel) of the converted probe. The eye opening is defined as the voltage difference between the minimum mark level and maximum space level for simulated result, or between three standard deviation down from mean mark level and up from mean space level for experimental result. From the simulation, the degradation of eye opening is within $2 \mathrm{~dB}$ for probe wavelength from $1500 \mathrm{~nm}$ to $1560 \mathrm{~nm}$. The degradation at the short wavelength end is due to walk-off of the signal from the probe in the HNL-DSF. Such degradation can be mitigated using dispersion-flattened nonlinear fiber, which enables wavelength conversion over $120 \mathrm{~nm}$ bandwidth [14]. This means delay range can be extended by simply replacing the probe amplifiers with wide band substitutes such as hybrid S/C-band EDFAs.

Fig. 7 shows the waveforms and the eye diagrams of the probe signal obtained at port 3 of the circulator in signal path obtained in experiment. When the pre-chirping signal is turned off, severe pulse distortion is observed from both the waveform and the eye diagram, where the mark level is hardly defined. It is noticeable that spikes are present at the pulse edges. The eye distortion and the presence of spike at pulse edges are due to GVD in fiber and residue phase modulation of the probe after XPolM. Given a normal dispersive fiber, optical pulse propagating along would usually experience pulse broadening even with SPM [20]. However, if the optical pulse is generated by a Kerr switch operating in inverting mode, the chirp induced on the pulse will be reverse of SPM-induced chirp as the chirp generated by a rising edge of the control pulse in Kerr switch will be transferred to the corresponding falling edge of the converted pulse. Fig. 8 shows the simulated waveforms of a Kerr switch-converted signal operating in inverting and noninverting modes. It is clear that while the signal pulse obtained from inverting Kerr switch contains spikes at the edges, the signal from noninverting Kerr switch suffers from severe ISI due to pulse broadening. As will be discussed later, operating the Kerr switch in inverting mode can improve the signal quality with pre-chirp.

By engaging pre-chirp, intensity fluctuation in mark level of probe signal eye is greatly suppressed as observable from Fig. 7. (a) Without Pre-chirp
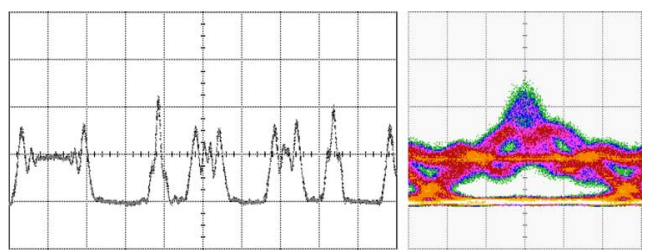

(b) With

Pre-chirp

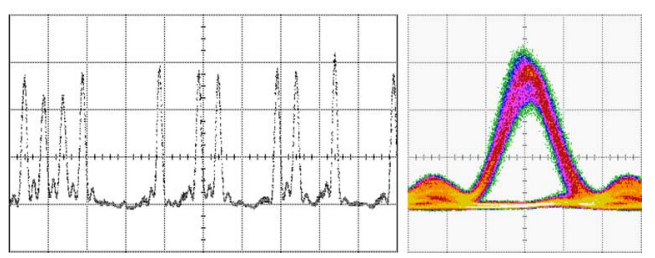

Fig. 7. Waveforms and eye diagrams of output probe when pre-chirp signal is (a) turned off and (b) turned on. Horizontal scales are $200 \mathrm{ps} / \mathrm{div}$ for waveforms and $20 \mathrm{ps} / \mathrm{div}$ for eye diagrams.

(a)
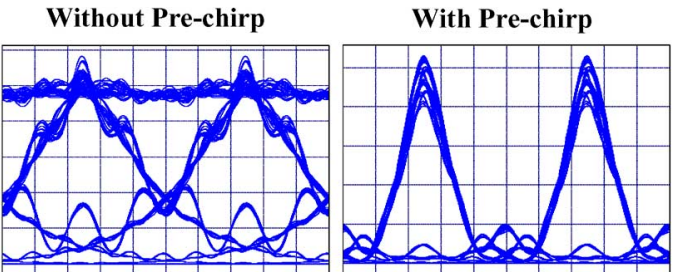

(b)

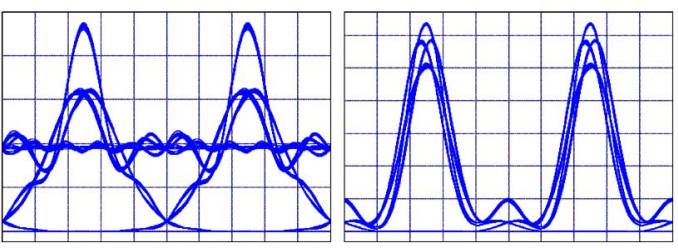

Fig. 8. Simulated eye diagrams of output probe with (a) noninverting and (b) inverting Kerr switches. Horizontal scale is 20 ps/div.

Quantitatively, the eye signal-to-noise ratio of the pulse is improved by $10 \mathrm{~dB}$. With strong chirp induced by pre-chirping technique, the weaker residue chirp due to XPM effect in Kerr switch is overridden. Therefore, each bit slot contains approximately equal amount of chirp. After the DCF, the probe pulse is compressed which the compressed pulse shape is determined by the chirp of the original pulse. As the amount of chirp is equal among the pulse, the pulse shape becomes approximately equal, and therefore results in intensity fluctuation reduction as in Fig. 7 and 8. When the eye of pre-chirped probe wave from inverting and noninverting converter is compared, intensity variation at mark and space level is greater for noninverting converter. This is because the residue chirp on the noninverting probe enhances the pulse broadening effect in the DCF with normal dispersion. The excessive pulse broadening renders the sinusoidal chirp on the probe wave inefficient for GVD compensation as the broadened pulse extends beyond the linear chirp region of the pulse. Therefore, ghost pulse is observable in the space level of the noninverting probe even with pre-chirp engaged.

In addition to intensity noise reduction, the intensity modulation of the probe wave is converted to RZ format with $30 \%$ duty cycle after the pre-chirp signal is turned on. However, pedestal pulse is clearly observed between the main pulses. The presence of pedestal pulse is caused by deviation of pre-chirping signal 

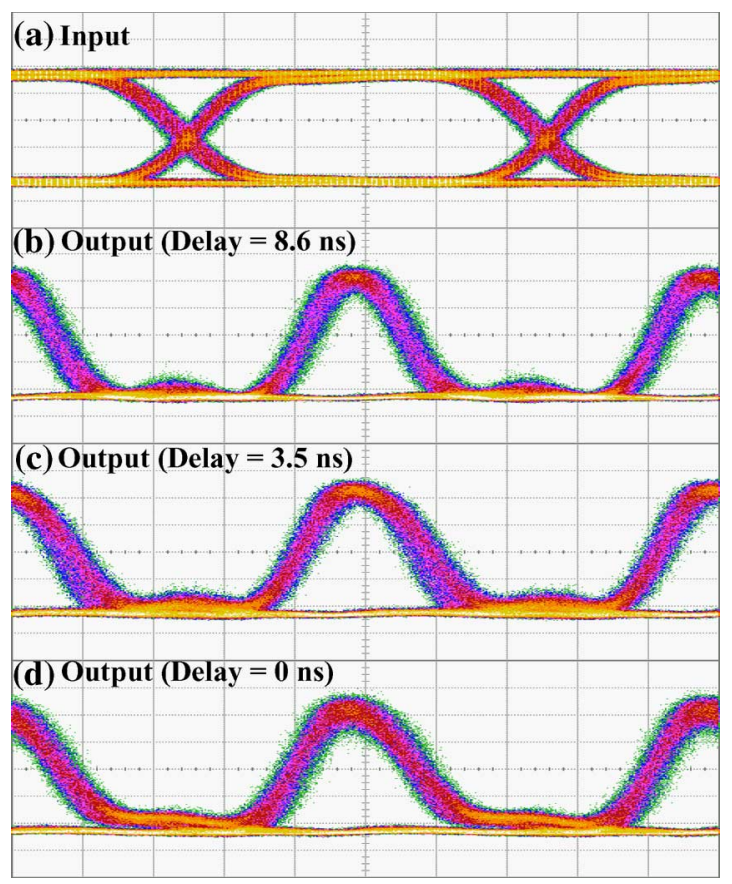

Fig. 9. Eye diagrams of input and output signal at different relative delay. Horizontal scale is $20 \mathrm{ps} / \mathrm{div}$.

used from the ideal one. Although linear chirp around the bit slot center is helpful in compensating second-order dispersion, the nonlinear chirp near to the bit slot emulates higher-order dispersion, which generates trailing pulses at both edges.

Fig. 9 shows the eye diagrams of the input NRZ signal, and the delayed RZ signal at port 3 of the circulator in signal path obtained in experiment. From the eye diagrams, clear eye opening is obtained throughout the delay range provided by the tunable delay. Thanks to the limiting transfer characteristic of the Kerr switch around the maximum transmission regime, the residue intensity modulation is suppressed to an unobservable level. The SNRs and extinction ratio of the output signals are over $21 \mathrm{~dB}$ and $15 \mathrm{~dB}$, which is comparable to or even surpass those of the input signal ( $25 \mathrm{~dB}$ and $13 \mathrm{~dB}$ respectively). Pedestal pulses are seen between adjacent signal pulses, which are inherited from the delayed probe wave. We believe the pedestal pulses can be eliminated by using common pedestal suppression techniques [23], [24]. Moreover, the pre-chirp conditions can be modified to achieve pedestal-free compression using the condition given in [21].

Fig. 10 shows the bit-error rate (BER) plot of the delayed RZ signals and the input NRZ signal. From the plot, error-free operation is achieved over the whole delay range provided by the tunable delay. The delayed signals have an uniform power penalty of $-1 \mathrm{~dB}$ at BER $=10^{-9}$. The apparent enhancement of receiver sensitivity of the delayed signals is due to difference in duty cycle between NRZ and RZ format, as RZ signal pulse has higher peak power when compared to its NRZ counterparts at the same average power level. With the difference in peak power level taken into account, the power penalty incurred by the tunable delay is $1.6 \mathrm{~dB}$ referencing to the typical sensitivity gain of $2.6 \mathrm{~dB}$ for $33 \%$ duty ratio RZ format [25]. The reduction of negative power penalty from typical sensitivity gain is believed to be

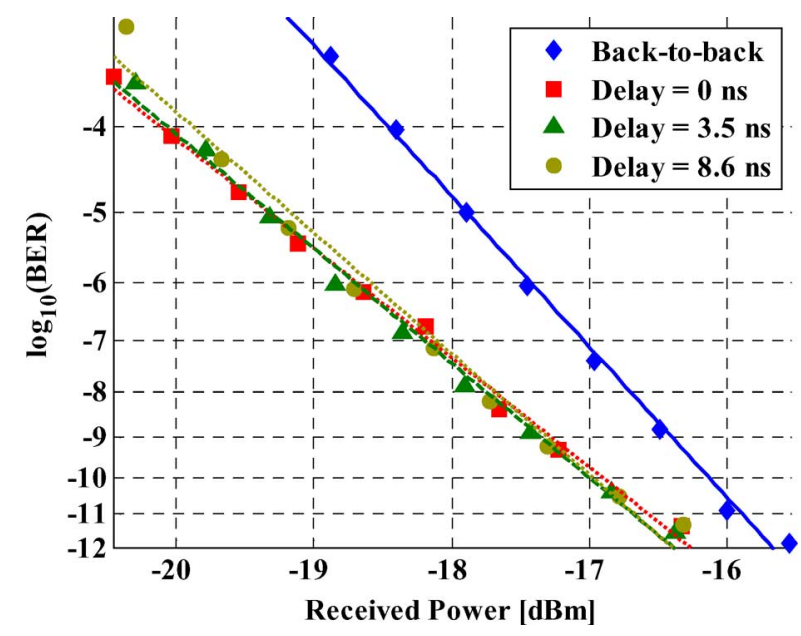

Fig. 10. BER plots of input signal, and delayed signal with $8.56 \mathrm{~ns}, 3.5 \mathrm{~ns}$ and 0 ns relative delay.

caused by three factors. Firstly, as the use of optical filters is kept minimal in the experimental setup, the excess ASE noise from EDFAs unavoidably affects the quality of the delayed probe and signal. Secondly, the presence of pesdestal pulse effectively decreases the peak power of the signal pulse when comparing to an ideal RZ signal, which results in apparent reduction in receiver sensitivity. Lastly, as the pulse duration of RZ signal is reduced, the adverse effect of timing jitter on receiver sensitivity is magnified. Nevertheless, the experimental results suggested the promising performance of the tunable delay over the whole delay range.

Finally, note that although Kerr switch wavelength converter in its simplest form only works with intensity-modulated signal, modification could be done on the Kerr switches to cope with phase-modulated input signals [26], [27]. This means the proposed scheme could be versatile regarding input signal modulation format.

\section{CONCLUSION}

We have demonstrated an optical delay with NRZ-RZ format conversion functionality based on optical Kerr switching and pulse pre-chirping technique. The wideband operating range of the Kerr switch wavelength converter enabled large delay tunable range up to $8.56 \mathrm{~ns}$. Meanwhile, limiting power transfer characteristic at maximum transmission regime of the Kerr switch helps to reduce the intensity fluctuation induced by GVD effect. To compensate GVD in fiber, pulse pre-chirp is deployed using phase modulation with sinusoidal signal, which also provides simultaneous NRZ-to-RZ format conversion functionality. With help from regenerative property of Kerr switch and pulse pre-chirping, $-1-\mathrm{dB}$ power penalty is achieved over the whole delay range. Further enhancement could be achieved by using optical amplifiers with larger gain bandwidth to increase tunable delay range, and by deploying simple pedestal removal measure to improve pulse shape.

\section{ACKNOWLEDGMENT}

The authors would like to acknowledge Sumitomo Electric Industries for providing the HNL-DSF used in this work. 


\section{REFERENCES}

[1] D. K. Hunter, M. C. Chia, and I. Andonovic, "Buffering in optical packet switches," J. Lightw. Technol., vol. 16, no. 12, pp. 2081-2094, Dec. 1998.

[2] I. Frigies and A. J. Seeds, "Optically generated true-time delay in phased array antennas," IEEE Trans. Microw. Theory Tech., vol. 43, no. 9, pp. 2378-2386, Sep. 1995.

[3] Y. Okawachi, M. S. Bigelow, J. E. Sharping, Z. Zhu, A. Schweinsberg, D. J. Gauthier, R. W. Boyd, and A. L. Gaeta," "Tunable all-optical delays via Brillouin slow light in an optical fiber," Phys. Rev. Lett., vol. 94, p. 153902, Apr. 2005.

[4] J. E. Sharping, Y. Okawachi, and A. L. Gaeta, "Wide bandwidth slow light using a Raman fiber amplifier," Opt. Exp., vol. 13, no. 16, pp. 6092-6098, Aug. 2005.

[5] D. Dahan and G. Eisenstein, "Tunable all optical delay via slow and fast light propagation in a Raman assisted fiber optical parametric amplifier: A route to all optical buffering," Opt. Exp., vol. 13, no. 16, pp. 6234-6249, Aug. 2005.

[6] L. Yi, W. Hu, Y. Su, M. Gao, and L. Leng, "Design and system demonstration of a tunable slow-light delay line based on fiber parametric process," IEEE Photon. Technol. Lett., vol. 18, no. 24, pp. 2575-2577, Dec. 2006

[7] L. Zhang, T. Luo, C. Yu, W. Zhang, and A. E. Willner, "Pattern dependence of data distortion in slow-light elements," J. Lightw. Technol., vol. 25, no. 7, pp. 1754-1760, Jul. 2007.

[8] Y. Okawachi, J. E. Sharping, C. Xu, and A. L. Gaeta, "Large tunable optical delays via self-phase modulation and dispersion," Opt. Exp., vol. 14 , no. 25 , pp. 12022-12027, Dec. 2006.

[9] J. Ren, N. Alic, E. Myslivets, R. E. Saperstein, C. J. Mckinstrie, R. M. Jopson, A. H. Gnauck, P. A. Andrekson, and S. Radic, "12.47 ns continuously-tunbale two-pump parametric delay," presented at the Proc. Eur. Conf. Opt. Commun. (ECOC) 2006, Cannes, France, Sep. 2001, paper Th 4.4.3, unpublished.

[10] J. E. Sharping, Y. Okawachi, J. van Howe, C. Xu, Y. Wang, A. E. Willner, and A. L. Gaeta, "All-optical, wavelength and bandwidth preserving, pulse delay based on parametric wavelength conversion and dispersion," Opt. Exp., vol. 13, no. 20, pp. 7872-7877, Oct. 2005.

[11] M. P. Fok and C. Shu, "Tunable optical delay using four-wave mixing in a $35-\mathrm{cm}$ highly nonlinear bismuth-oxide fiber and group velocity dispersion," J. Lightw. Technol., vol. 25, no. 5, pp. 499-504, Mar. 2008.

[12] N. Alic, J. R. Windmiller, J. B. Coles, and S. Radic, "Two-pump parametric optical delays," IEEE J. Sel. Topics Quantum Electron., vol. 14, no. 3, pp. 681-690, May-Jun. 2008.

[13] L. Christen, I. Fazal, O. F. Yilmaz, X. Wu, S. Nuccio, A. E. Willner, C. Langrock, and M. M. Fejer, "Tunable 105-ns optical delay for 80-Gbit/s RZ-DQPSK, 40-Gbit/s RZ-DPSK, and 40-Gbit/s RZ-OOK signals using wavelength conversion and chromatic dispersion," presented at the Proc. Opt. Fiber Comm. Conf., San Diego, CA, Feb. 2008, paper OTuD1, unpublished.

[14] C. H. Kwok, C. W. Chow, H. K. Tsang, C. Lin, and A. Bjarklev, "Nonlinear polarization rotation in a dispersion-flattened photonic-crystal fiber for ultrawideband ( $>100 \mathrm{~nm}$ ) all-optical wavelength conversion of $10 \mathrm{Gbit} / \mathrm{s}$ nonreturn-to-zero signals," Opt. Lett., vol. 31, no. 12, pp. 1782-1784, Jun. 2006.

[15] J. van Howe and C. Xu, "Ultrafast optical signal processing based upon space-time dualities," J. Lightw. Technol., vol. 24, no. 7, pp. 2649-2662, Jul. 2006

[16] S. Kumar, "Analysis of degenerate four-wave-mixing noise in return-to-zero optical transmission systems including walk-off," $J$. Lightw. Technol., vol. 23, no. 1, pp. 310-320, Jan. 2005.

[17] B. P. P. Kuo, P. C. Chui, and K. K. Y. Wong, "A comprehensive study on crosstalk suppression techniques in fiber optical parametric amplifier by modulation format," IEEE J. Sel. Topics Quantum Electron., vol. 14, no. 3, pp. 659-665, May-Jun. 2008.

[18] N. Alic, R. M. Jopson, J. Ren, E. Myslivets, R. Jiang, A. H. Gnauck, and S. Radic, "Impairments in deeply-saturated optical parametric amplifiers for amplitude- and phase-modulated signals," Opt. Exp., vol. 15, no. 14, pp. 8997-9008, Jul. 2007.

[19] B. P. P. Kuo, P. C. Chui, and K. K. Y. Wong, "Simultaneous optical delay and NRZ-RZ format conversion via cross-polarization modulation and pulse pre-chirping," presented at the Proc. Conf. Laser and Electro-Optics (CLEO) 2008, San Jose, May 2008, paper CTuB2, unpublished.
[20] G. P. Agrawal, Nonlinear Fiber Optics, 3rd ed. San Diego, CA: Academic, 2001

[21] T. Komukai, T. Yamamoto, and S. Kawanishi, "Optical pulse generator using phase modulator and linearly chirped fiber Bragg gratings," IEEE Photon. Technol. Lett., vol. 17, no. 8, pp. 1746-1748, Aug. 2005.

[22] J. H. Lee, T. Nagashima, T. Hasegawa, S. Ohara, N. Sugimoto, and K. Kikuchi, "Wide-band tunable wavelength conversion of $10 \mathrm{~Gb} / \mathrm{s}$ nonreturn-to-zero signal using cross-phase-modulation-induced polarization rotation in 1-m bismuth oxide-based nonlinear optical fiber," IEEE Photon. Technol. Lett., vol. 18, no. 1, pp. 298-300, Jan. 2006.

[23] K. Smith, N. J. Doran, and P. G. J. Wigley, "Pulse shaping, compression and pedestal suppression employing a nonlinear-optical loop mirror," Opt. Lett., vol. 15, no. 22, pp. 1294-1296, Nov. 1990.

[24] N. Nishizawa and A. Murayama, "Pedestal suppression of ultrashort pulses by using a birefringent nonlinear polarization rotation mirror," Opt. Lett., vol. 32, no. 24, pp. 3516-3518, Dec. 2007.

[25] P. J. Winzer and A. Kalmar, "Sensitivity enhancement of optical receivers by impulsive coding," J. Lightw. Technol., vol. 17, no. 2, pp. 171-177, Feb. 1999.

[26] L. Moeller, Y. Su, C. Xie, R. Ryf, C. R. Doerr, X. Liu, and L. L. Buhl, "Generation of a 160-Gb/s RZ-DPSK signal and its detection with a one-bit Mach-Zehnder interferometer," presented at the Proc. Eur. Conf. Opt. Commun. (ECOC) 2004, Stockholm, Sweden, 2004, paper Th4.4.6, unpublished.

[27] E. S. Awad, "A technique for rephrasing $+2 \mathrm{R}$ regeneration of $40-\mathrm{Gb} / \mathrm{s}$ NRZ-DPSK data," IEEE Photon. Technol. Lett., vol. 20, no. 10, pp. 848-850, May 2008.

Bill Ping-Piu Kuo (S'07) received the B.Eng. degree (with first-class honors) in electronic and communications engineering from the University of Hong Kong, Hong Kong, in 2006, where he is currently working toward the M.Phil. degree in electronic engineering.

His research interests include fiber-optic parametric and Kerr effects and their applications in optical and microwave communication systems.

P. C. Chui, photograph and biography not available at the time of publication.

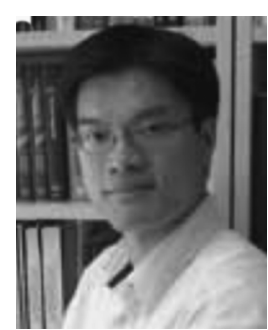

Kenneth Kin-Yip Wong (M'03) received the B.E. degree (first class honors with medal award) in electrical engineering and the B.S. degree in physics from the University of Queensland, Brisbane, Australia, in 1997, and the M.S. and Ph.D. degrees in electrical engineering from Stanford University, Stanford, CA, in 1998 and 2003, respectively.

He was a member of the Photonics and Networking Research Laboratory, Stanford University. His field of research included DWDM systems, SCM optical systems, fiber nonlinearity, fiber-optical parametric amplifiers, and photonic crystal fibers. He is the author or coauthor of over 100 journal and conference papers. He was with Hewlett-Packard Laboratories as a Research Engineer and contributed to projects that included parallel optics and VCSEL in 1998 and 1999. He was also an independent consultant to Innovation CORE (A Sumitomo Electric Company), CA, in 2004. He is currently an Assistant Professor with the Department of Electrical and Electronic Engineering, University of Hong Kong.

Dr. Wong is a member of the Optical Society of America (OSA), SPIE, and the IEEE Lasers and Electro-Optic Society (LEOS). He was the recipient of the Optical SOSA New Focus Student Award and the IEEE/LEOS Graduate Student Fellowship, both in 2003. He was the recipient of Best Teacher Award 2005-2006 at the University of Hong Kong. He is a reviewer for Optics Letters, Journal of the Optical Society of America B, Optics Express, IEEE PHOTONICS TECHNOLOGY LeTters, the JOURNAL OF LightwaVE TECHNOLOGY, Electronics Letters, and Optics Communications. 